This item was submitted to Loughborough's Research Repository by the author.

Items in Figshare are protected by copyright, with all rights reserved, unless otherwise indicated.

\title{
Rotational vibration measurements using laser doppler vibrometry: comprehensive theory and practical application
}

PLEASE CITE THE PUBLISHED VERSION

http://dx.doi.org/10.1006/jsvi.2000.3134

\section{PUBLISHER}

Elsevier @ Academic Press

VERSION

AM (Accepted Manuscript)

\section{LICENCE}

CC BY-NC-ND 4.0

\section{REPOSITORY RECORD}

Bell, John R., and Steve Rothberg. 2019. "Rotational Vibration Measurements Using Laser Doppler Vibrometry: Comprehensive Theory and Practical Application”. figshare. https://hdl.handle.net/2134/9664. 
This item was submitted to Loughborough's Institutional Repository (https://dspace.lboro.ac.uk/) by the author and is made available under the following Creative Commons Licence conditions.

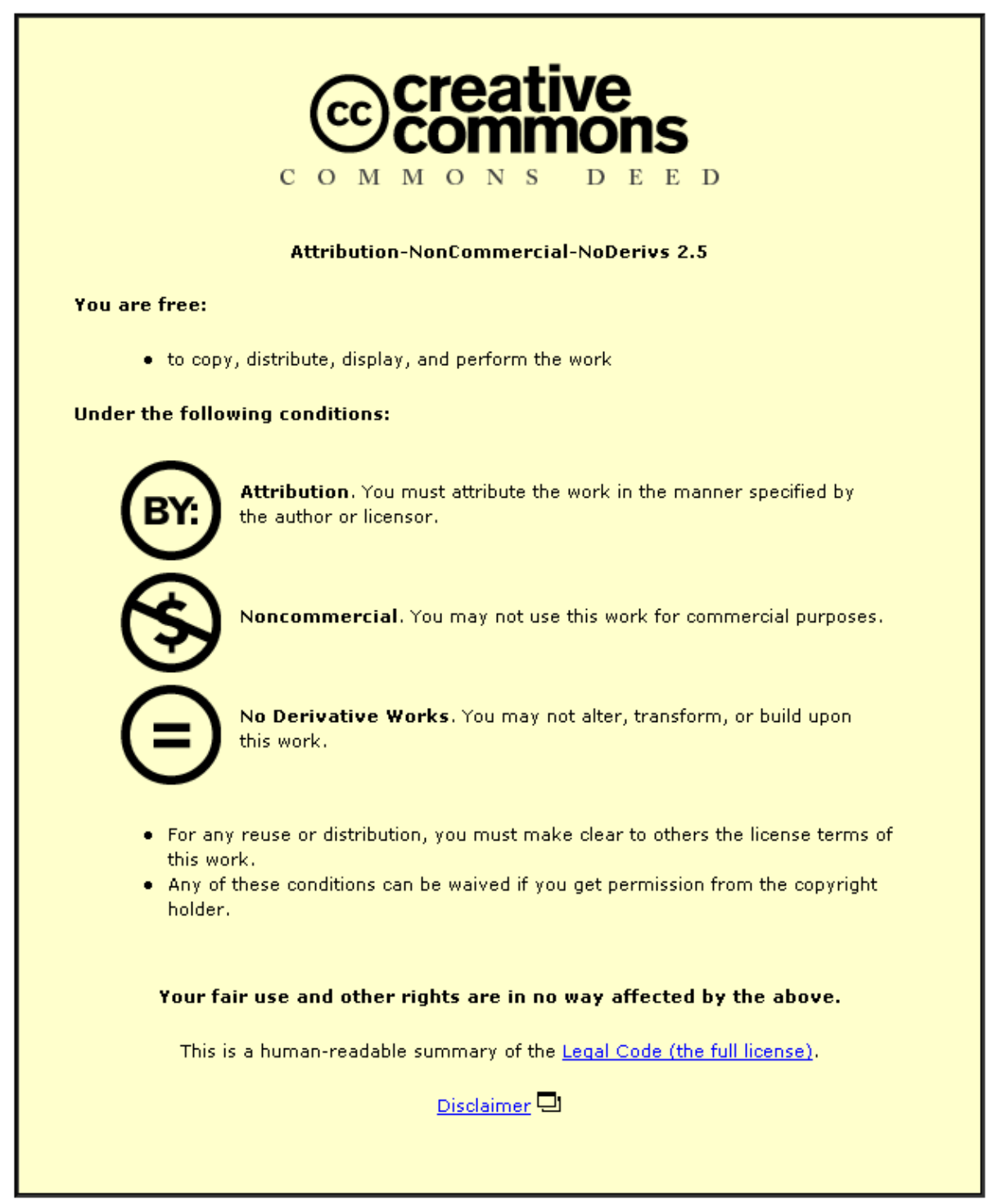

For the full text of this licence, please go to: http://creativecommons.org/licenses/by-nc-nd/2.5/ 


\title{
ROTATIONAL VIBRATION MEASUREMENTS USING LASER \\ DOPPLER VIBROMETRY: COMPREHENSIVE THEORY AND \\ PRACTICAL APPLICATION
}

\author{
J. R. BELL and S. J. ROTHBERG \\ Department of Mechanical Engineering, Loughborough University \\ Loughborough, Leicestershire, UK
}

Total Number of Main Text Pages: 24

Total Number of Figures: 10 in 14 pieces

Short Title: ROTATIONAL VIBRATION MEASUREMENTS USING LDV

Address for Correspondence:

Dr S. J. Rothberg

Department of Mechanical Engineering

Loughborough University

LOUGHBOROUGH

Leicestershire LE11 3TU 


\section{SUMMARY}

This paper builds on a previous study of the velocity sensed by a single laser vibrometer beam incident on a target in an arbitrary direction to predict the velocity sensed by multiple laser beams with arbitrary orientations. The usefulness of parallel beam arrangements is demonstrated and a concise new theory for the difference velocity sensitivity for two parallel beams is presented. Parallel beam arrangements for virtually unambiguous measurement of torsional vibration are presented along with arrangements that allow measurement of the pitch and yaw vibration to be derived. Resolution of individual pitch and yaw motions is shown not to be possible by any geometrical arrangement of the beams but a post-processing technique that enables the genuine pitch and yaw vibrations to be estimated from the measurements of the pitch and yaw vibration sets is demonstrated and validated experimentally in the laboratory. Measurements of pitch and yaw vibration are, for the first time, obtained from the crankshaft of a running diesel engine, enabling identification of the first natural frequency of the crankshaft in bending. 


\section{INTRODUCTION}

The principle of Laser Doppler Vibrometry (LDV) relies on the detection of the Doppler frequency shift in coherent light scattered from a moving target. By measuring the frequency shift, a time resolved measurement of the target velocity is made. The non-contact nature of Laser Vibrometers offers significant advantages over traditional contacting vibration transducers and measurements on hot, light or rotating components are often cited as important applications [1].

For rotational or angular vibrations, a non-contact transducer capable of measuring directly from any location on a structure, especially a rotor, would be a valuable asset and LDV offers this possibility. A variety of optical configurations have been proposed for angular vibration measurement on non-rotating structures. These include angular measurements derived from differential measurements [2], measurement of the change in position of a reflected laser beam [3] and continuously scanning regimes [4].

A parallel beam arrangement has been developed [5] and is available commercially for torsional vibration measurement on rotors. Torsional vibrations are often a source of problems in rotating machinery and can result in increased component wear, shaft cracking and fatigue. This parallel beam arrangement can also be used for measurement of other angular vibration components, either on rotating or non-rotating structures. One example application would be measurement of bending vibrations in the crankshafts of automotive engines which are thought to be linked with engine "rumble” [6], an unpleasant noise heard during acceleration. An investigation of the velocity sensed by a single laser beam incident on a rotating shaft vibrating in all six degrees-of-freedom [7] revealed that the measured velocity is made up of six separate vibration "sets", each a combination of motion parameters. Using a single laser vibrometer it is possible to isolate the translational vibration "sets" (two radial and one axial) but it was shown not to be possible to isolate the three rotational vibration "sets", those 
including torsional and bending vibration. This paper extends this first analysis by concentrating on multiple laser beam configurations that enable the rotational vibration "sets" to be measured. The particular usefulness of parallel beam arrangements is demonstrated and a concise, new theory is given to describe the difference velocity sensitivity for such an arrangement. The results of this analysis offer the engineer a valuable tool for defining the velocity sensed in any proposed measurement configuration.

Previous experience with LDV measurements made on rotors has highlighted how measurements can be ambiguous, affected by rotational vibration components perpendicular to the component it is intended to measure. Earlier studies have acknowledged crosssensitivities in torsional vibration measurements [8] for simplified target motions and the theory here confirms the conclusions of these earlier studies but additionally shows the effects of all vibration components of the arbitrary motion.

Measurements are then made on the crankshaft of a running diesel engine with the intention of resolving pitch and yaw vibration from simultaneous measurements which individually show cross-sensitivity to pitch in an intended yaw measurement and vice-versa. Using a postprocessing technique, initially developed to resolve the cross-sensitivity in radial vibration measurements [9], these measurements are processed to give unambiguous estimation of nonsynchronous pitch and yaw vibration.

\section{TOTAL DIFFERENCE VELOCITY MEASURED BY PAIRS OF LASER BEAMS}

\subsection{VELOCITY MEASURED BY A SINGLE LASER BEAM INCIDENT ON A ROTATING SHAFT}

A thorough analysis of this case has been presented previously [7]. As shown in Figure 1, the case considered is that of a rotating shaft, which can have arbitrary shape, undergoing an 
arbitrary vibration requiring three translational and three rotational co-ordinates for description. If the illuminated axial element of the shaft can be assumed to be rigid, the velocity measured by a laser beam, orientated according to the angles $\alpha$ and $\beta$ (refer to Figure 2) and incident on a rotating shaft, is given by:

$$
\begin{aligned}
U_{m} & =\cos \beta \cos \alpha\left[V_{x}+\left(\dot{\theta}_{z}+\Omega\right) a_{y}-\left(\dot{\theta}_{y}-\Omega \theta_{x}\right) a_{z}\right] \\
& +\cos \beta \sin \alpha\left[V_{y}-\left(\dot{\theta}_{z}+\Omega\right) a_{x}+\left(\dot{\theta}_{x}+\Omega \theta_{y}\right) a_{z}\right] \\
& -\sin \beta\left[V_{z}-\left(\dot{\theta}_{x}+\Omega \theta_{y}\right) a_{y}+\left(\dot{\theta}_{y}-\Omega \theta_{x}\right) a_{x}\right] \\
& -\left(y_{0} \sin \beta+z_{0} \cos \beta \sin \alpha\right)\left[\dot{\theta}_{x}+\Omega \theta_{y}\right] \\
& +\left(z_{0} \cos \beta \cos \alpha+x_{0} \sin \beta\right)\left[\dot{\theta}_{y}-\Omega \theta_{x}\right] \\
& +\left(x_{0} \cos \beta \sin \alpha-y_{0} \cos \beta \cos \alpha\right)\left[\dot{\theta}_{z}+\Omega\right]
\end{aligned}
$$

where $\left(V_{x}, V_{y}, V_{z}\right)$ and $\left(a_{x}, a_{y}, a_{z}\right)$ are the translational vibration velocities and displacements of the origin $\mathrm{O}$ in the $(x, y, z)$ directions, $\Omega$ is the total rotation speed of the axial shaft element (combining shaft rotation speed and any torsional vibration or speed fluctuation of the axial element), $\left(\dot{\theta}_{x}, \dot{\theta}_{y}, \dot{\theta}_{z}\right)$ are the angular vibration velocities of the shaft around the $(x, y, z)$ axes, referred to as pitch, yaw and roll respectively, and $\left(x_{0}, y_{0}, z_{0}\right)$ is the position of an arbitrary known point that lies along the line of the beam. It is usual to take $\left(x_{0}, y_{0}, z_{0}\right)$ as the initial point of incidence on the structure at set-up, in which case the point might be referred to as lying in the "measurement plane”.

Equation (1) allows the vibration engineer to be sure of vibration component sensitivity for any laser beam arrangement on any target, including a rotating shaft. It shows that the measured velocity is the sum of six terms, each the product of a combination of geometric parameters and a combination of motion parameters - the vibration "sets". The six vibration 
sets, shown in square brackets, are inseparable combinations of different motion parameters. This is an important result because it shows that, however a laser beam is aligned, only the combinations of motion parameters within the square brackets can be measured directly.

Throughout the remainder of the discussion, the six vibration sets in equation (1) will be referred to by the vibration parameter in each group that might be regarded as the intended measurement. These are, in the order that they are written above in equation (1), the $x$ radial, $y$ radial, axial, pitch and yaw vibration sets and the rotation speed set which includes torsional vibration.

Equation (1) can be simplified by setting $z_{0}=0$ so that the plane of the origin of the $x y z$ axes and the "measurement plane" are coincident, since this is just a matter of definition. Isolation of any one of the six sets then requires appropriate choice of values for $\alpha, \beta, x_{0}$ and $y_{0}$. Values for these geometric coefficients can be found that enable the radial and axial vibration sets to be isolated and this was set out in a preceding publication [7]. Isolation of the rotational vibration sets requires the geometric coefficients of the three translational vibration sets to equal zero i.e. $\cos \beta \cos \alpha=\cos \beta \sin \alpha=\sin \beta=0$, to which there is no solution. This means that none of the rotational vibration sets can be isolated using a single laser beam.

\subsection{MEASUREMENT OF ROTATIONAL VIBRATION SETS USING MULTIPLE LASER BEAMS}

The next logical step to take in attempting to measure the rotational vibration sets is to use two laser beams. In this section the particular usefulness of a pair of parallel beams will be demonstrated. In an interferometer it is the difference velocity that can be obtained most conveniently by heterodyning and so it is the difference velocity that will be analysed in this section. A sum velocity could effectively be obtained by re-orientating one of the incident beams by $180^{\circ}$ and so, by examining the difference velocity for arbitrarily orientated beams, 
all possible eventualities can be explored. Using equation (1) and assuming that the shaft is rigid between the axial elements probed by each beam, the difference in the velocity measured by two beams $\Delta U_{m}=U_{m 1}-U_{m 2}$ can be written as:

$$
\begin{aligned}
\Delta U_{m} & =\left(\cos \beta_{m 1} \cos \alpha_{m 1}-\cos \beta_{m 2} \cos \alpha_{m 2}\right)\left[V_{x}+\left(\dot{\theta}_{z}+\Omega\right) a_{y}-\left(\dot{\theta}_{y}-\Omega \theta_{x}\right) a_{z}\right] \\
& +\left(\cos \beta_{m 1} \sin \alpha_{m 1}-\cos \beta_{m 2} \sin \alpha_{m 2}\right)\left[V_{y}-\left(\dot{\theta}_{z}+\Omega\right) a_{x}+\left(\dot{\theta}_{x}+\Omega \theta_{y}\right) a_{z}\right] \\
& -\left(\sin \beta_{m 1}-\sin \beta_{m 2}\right)\left[V_{z}-\left(\dot{\theta}_{x}+\Omega \theta_{y}\right) a_{y}+\left(\dot{\theta}_{y}-\Omega \theta_{x}\right) a_{x}\right] \\
& -\left(y_{0 m 1} \sin \beta_{m 1}-y_{0 m 2} \sin \beta_{m 2}+z_{0 m 1} \cos \beta_{m 1} \sin \alpha_{m 1}-z_{0 m 2} \cos \beta_{m 2} \sin \alpha_{m 2}\right)\left[\dot{\theta}_{x}+\Omega \theta_{y}\right] \\
& +\left(x_{0 m 1} \sin \beta_{m 1}-x_{0 m 2} \sin \beta_{m 2}+z_{0 m 1} \cos \beta_{m 1} \cos \alpha_{m 1}-z_{0 m 2} \cos \beta_{m 2} \cos \alpha_{m 2}\right)\left[\dot{\theta}_{y}-\Omega \theta_{x}\right] \\
& +\left(x_{0 m 1} \cos \beta_{m 1} \sin \alpha_{m 1}-y_{0 m 1} \cos \beta_{m 1} \cos \alpha_{m 1}\right. \\
& \left.-x_{0 m 2} \cos \beta_{m 2} \sin \alpha_{m 2}+y_{0 m 2} \cos \beta_{m 2} \cos \alpha_{m 2}\right)\left[\dot{\theta}_{z}+\Omega\right]
\end{aligned}
$$

where the subscripts ' $\mathrm{m} 1$ ' and ' $\mathrm{m} 2$ ' for the geometric parameters denote the particular beam. At this stage it can be noted that, in measurement circumstances where it is not possible to assume that the shaft is rigid between the axial elements probed by each beam, the approach to describe the difference in the velocity measured by the two beams would be also to denote the vibration sets in equation (1) according to the particular beam. Such a situation might be where one beam is incident on a vibrating turbine blade and the second is incident on the relatively rigid disc to which the blades are fixed.

Returning to the situation described by equation (2) and the issue of isolating one of the six vibration sets, it is necessary to choose geometric parameters that make the coefficients of the remaining vibration sets equal to zero while having a non-zero coefficient for the desired set. To measure one of the rotational vibration sets successfully, the coefficients of the radial and axial vibration sets must always be zero. Solving the following three simultaneous equations: 
$\cos \beta_{m 1} \cos \alpha_{m 1}=\cos \beta_{m 2} \cos \alpha_{m 2}$

$\cos \beta_{m 1} \sin \alpha_{m 1}=\cos \beta_{m 2} \sin \alpha_{m 2}$

$\sin \beta_{m 1}=\sin \beta_{m 2}$

gives solutions of $\beta_{m 1}=\beta_{m 2}=\beta$ and $\alpha_{m 1}=\alpha_{m 2}=\alpha$. Geometrically these solutions equate to the beams being parallel and oriented in the same direction for the differential measurement or in opposite directions for a sum velocity derivation. In this latter case, the beams would be incident on opposite sides of the shaft which would usually make for an inconvenient optical arrangement. In either case, it can be concluded that to make a measurement immune to both axial and radial vibrations, parallel beams are required. For this reason, this paper will concentrate on the use of parallel beams. In practice it is possible to use two separate interferometers and obtain the difference in outputs electronically [10] or to combine beams and find the difference velocity optically as in the first arrangement proposed for torsional vibration measurement, the Laser Torsional Vibrometer (LTV) [5].

Cross-beam arrangements have been popular in the past for torsional vibration measurement [11] and they have also been used to determine the centre of rotation of rotating objects [12]. They differ from the arrangements described here in the method of light collection but the vibration sets to which measurements are sensitive can be investigated using the theory presented in this paper by considering the difference in velocity measured by two single laser beams at an angle to one another, with the light scattered from each incident point collected in direct back-scatter. Figure 3 shows the comparable arrangement of the beams for a torsional vibration measurement with both beams lying in the $x y$ plane. Using equation (2) the difference in the velocity measured by the two single beams is:

$$
\Delta U_{m}=2 \sin \alpha\left[V_{y}+\left(\dot{\theta}_{z}+\Omega\right)\left(x_{0}-a_{x}\right)+\left(\dot{\theta}_{x}+\Omega \theta_{y}\right) a_{z}\right]
$$


where $\alpha$ is the half-angle between the two beams and the intersection of the beams at $\left(x_{0}, y_{0}, z_{0}\right)$ is considered as the known point for both beams. As expected, the velocity measured includes radial, axial, pitch and yaw components in addition to the desired torsional vibration component. The recognised sensitivity to $V_{y}$ provided much of the motivation for the development of the LTV. It is interesting to note that in this configuration it is not necessary for the beams to cross on the target surface for equation (4) to hold. In the usual cross-beam configuration the method of light collection made this important. Light was collected in any direction but in the same direction for each beam allowing detection at any convenient location. Compared to equation (4) the geometric coefficient was reduced by a factor of two but the velocity component sensitivity (within the square brackets) was the same. The reported use to determine the centre of rotation relied on minimising the mean

value of $\left(\dot{\theta}_{z}+\Omega\right) x_{0}$ and experience has indicated that this is an acceptable approach. Error in this process is incurred, however, by the presence of products of oscillatory terms with the same frequency introducing signal components with non-zero means.

In the next section the difference velocity measured by a pair of parallel laser beams with arbitrary orientation and incident on a shaft undergoing arbitrary motion will be examined.

\subsection{CONCISE THEORY FOR THE DIFFERENCE VELOCITY MEASURED BY A PAIR OF PARALLEL LASER BEAMS}

The versatile approach developed previously [7] relied on the observation that although the point of incidence of the laser beam on the target might change position in space, it would always lie somewhere along the line of the beam itself. This simplified prediction of the vibrometer velocity sensitivity significantly compared to previous analyses and led to the 
derivation of equation (1) from which equation (2) followed. The same approach will be applied to this two beam arrangement, even though it is possible to manipulate equation (2), because the resulting derivation is more concise and comprehensive than any previous analysis.

The points $\mathrm{P}_{1}$ and $\mathrm{P}_{2}$, defined by the position vectors $\vec{r}_{P 1}$ and $\vec{r}_{P 2}$, are the points in space where the lines of the laser beams intersect the surface of the shaft. The velocities of $\mathrm{P}_{1}$ and $\mathrm{P}_{2}, \vec{V}_{P 1}$ and $\vec{V}_{P 2}$, are the sums of the translational velocity of origin $\mathrm{O}, \vec{V}_{0}$, and the velocity of each point relative to $\mathrm{O}$ as a result of rotation about an instantaneous rotation axis passing through $\mathrm{O}$ at angular velocity $\vec{\omega}$ :

$$
\begin{aligned}
& \vec{V}_{P 1}=\vec{V}_{o}+\left(\vec{\omega} \times \vec{r}_{P 1}\right) \\
& \vec{V}_{P 2}=\vec{V}_{o}+\left(\vec{\omega} \times \vec{r}_{P 2}\right)
\end{aligned}
$$

As the shaft vibrates and rotates the positions of the points of incidence, not only on the target but also in space, will change continuously especially if the target has a non-circular crosssection. As Figure 4 shows, the changed positions of the incident points at any arbitrary instant of time are now written as:

$$
\begin{aligned}
& \vec{r}_{P 1}=\left[\vec{r}_{01}-\vec{A}\right]+p_{1} \hat{b} \\
& \vec{r}_{P 2}=\left[\vec{r}_{02}-\vec{A}\right]+p_{2} \hat{b}
\end{aligned}
$$

where $\vec{r}_{01}$ and $\vec{r}_{02}$ are the (constant) position vectors for known points on the line of each beam, $\vec{A}$ is the (time-varying) translational vibration displacement of the origin and $p_{1}$ and $p_{2}$ are (time-varying) unknown quantities that are used to account for changes in the points of 
incidence due to target shape and vibration. $\hat{b}$ is the unit vector defining the direction of both beams since they are parallel.

The difference in the velocity measured by the two beams, $\Delta U_{m}$, is the difference in the component of the velocity of the incident points in the direction of the incident beam. Assuming that $\vec{\omega}$ is maintained across the probed region (i.e. the shaft remains rigid across the probed region), by substituting equations (6a\&b) into equations (5a\&b), the difference velocity measured between the two beams is:

$$
\Delta U_{m}=\hat{b} \cdot\left(\vec{V}_{P 1}-\vec{V}_{P 2}\right)=\hat{b} \cdot\left[\vec{\omega} \times\left(\vec{r}_{01}-\vec{r}_{02}\right)+\vec{\omega} \times\left(p_{1}-p_{2}\right) \hat{b}\right]
$$

Inspection of equation (7) shows that the second scalar triple product can be re-arranged to $\left(p_{1}-p_{2}\right) \vec{\omega} \cdot(\hat{b} \times \hat{b})$ which will always be zero, revealing that the measurement is always insensitive to any variation in the shape of the shaft. (Note that "shape" variation, as used in this paper, refers to variations in shaft dimensions and not to any dynamic shape variation). Equation (7) also demonstrates the immunity to translational motion offered by the parallel beam arrangement. The first scalar triple product in equation (7) is dependent on the difference in position of known points on the line of each beams. Since the choice of known position is arbitrary, the difference in position can be chosen so that $\vec{r}_{01}-\vec{r}_{02}=\vec{d}$ where $\vec{d}$ is a vector in the plane of the beams perpendicular to $\hat{b}$ and of magnitude equal to the perpendicular separation of the beams, $d$. The measured velocity can therefore be written simply as:

$$
\Delta U_{m}=\vec{\omega} \cdot(\vec{d} \times \hat{b})
$$


In order to make equation (8) of more direct practical use, $\hat{b}$ and $\vec{d}$ need to be described in terms of measurable parameters. Figure 2 shows that for a beam orientated according to the angles $\alpha$ and $\beta$ :

$\hat{b}=\cos \beta \cos \alpha \hat{x}+\cos \beta \sin \alpha \hat{y}-\sin \beta \hat{z}$

For $\vec{d}$, consider an initial position in the $y z$ plane inclined at an angle, $\gamma$, to $\hat{z}$ as shown in Figure 5. After finite rotations first by $\beta$ and then by $\alpha, \vec{d}$ becomes:

$$
\vec{d}=d[(\cos \gamma \sin \beta \cos \alpha+\sin \gamma \sin \alpha) \hat{x}+(\cos \gamma \sin \beta \sin \alpha-\sin \gamma \cos \alpha) \hat{y}+(\cos \gamma \cos \beta) \hat{z}]
$$

This is easily obtained by repeated application of the transformation [13]:

$$
\vec{r}^{\prime}=\vec{r}+(1-\cos \rho)[\hat{n} \times(\hat{n} \times \vec{r})]+\sin \rho(\hat{n} \times \vec{r})
$$

which describes the new position, $\vec{r}^{\prime}$, of a vector, $\vec{r}$, following a rotation, $\rho$, about an axis defined by the unit vector, $\hat{n}$. The three rotations $\gamma, \beta$ and $\alpha$ are finite and, therefore, this order of rotation must be maintained.

Angular velocity can also be expanded into its components [7]:

$$
\vec{\omega}=\left(\dot{\theta}_{x}+\Omega \theta_{y}\right) \hat{x}+\left(\dot{\theta}_{y}-\Omega \theta_{x}\right) \hat{y}+\left(\dot{\theta}_{z}+\Omega\right) \hat{z}
$$


which accounts for the change in direction of the shaft spin axis as the shaft tilts. Substituting equations (9a,b\&d) into equation (8) enables the measured difference velocity to be written as:

$$
\begin{aligned}
\Delta U_{m} & =d\left[(\sin \gamma \sin \beta \cos \alpha-\cos \gamma \sin \alpha)\left[\dot{\theta}_{x}+\Omega \theta_{y}\right]\right. \\
& \left.+(\sin \gamma \sin \beta \sin \alpha+\cos \gamma \cos \alpha)\left[\dot{\theta}_{y}-\Omega \theta_{x}\right]+\sin \gamma \cos \beta\left[\dot{\theta}_{z}+\Omega\right]\right]
\end{aligned}
$$

which is consistent with equation (2), simplified according to the parallel beam arrangement under consideration. Equation (10a) also conveniently describes the difference velocity directly in terms of the perpendicular beam separation rather than the individual positions $\left(x_{0 m 1}, y_{0 m 1}, z_{0 m 1}\right)$ and $\left(x_{0 m 2}, y_{0 m 2}, z_{0 m 2}\right)$.

If the subtraction of the velocities is performed optically then it is, in fact, the modulus of the difference velocity that is measured. In addition, a practical optical configuration might include frequency shifts in one or both beams and this is the case for the novel configuration used in section 4. If shifts are included then the measured difference velocity is:

$$
\begin{aligned}
\Delta U_{m} & =\mid \frac{\lambda}{2}\left[f_{1}-f_{2}\right]+d\left[(\sin \gamma \sin \beta \cos \alpha-\cos \gamma \sin \alpha)\left[\dot{\theta}_{x}+\Omega \theta_{y}\right]\right. \\
& \left.+(\sin \gamma \sin \beta \sin \alpha+\cos \gamma \cos \alpha)\left[\dot{\theta}_{y}-\Omega \theta_{x}\right]+\sin \gamma \cos \beta\left[\dot{\theta}_{z}+\Omega\right]\right]
\end{aligned}
$$

where $\lambda$ is the laser wavelength and $f_{1}$ and $f_{2}$ are the frequency shifts in beams one and two respectively. The shifts enable direction discrimination in the measured difference velocity. For brevity, the analysis will continue with acknowledgement of the ability to discriminate direction but without explicit inclusion of the shifts. 
Isolation of the rotation speed set requires $\beta=0$ and $\gamma=\frac{\pi}{2}$ so that:

$\Delta U_{m}=d\left[\dot{\theta}_{z}+\Omega\right]$

This shows that in order to make measurements of rotation speed, including torsional vibration, with immunity to other angular vibrations, the beams must be orientated perpendicular to the shaft rotation axis at any angle $\alpha$. A previous study [8] highlighted the sensitivity of measurements made with the Laser Torsional Vibrometer to angular vibrations and assessed this effect on a typical measurement when $\beta \neq 0$.

Simultaneous measurements of the rotation speed set at two different axial locations on a flexible shaft can be used to provide a real-time measurement of shaft twist. This can then provide a measurement of shaft torque using the linear relationship between twist and torque.

Isolation of the pitch and yaw vibration sets requires either $\gamma=\frac{\pi}{2}$ and $\beta= \pm \frac{\pi}{2}$, where the beams are incident on the end faces of the shaft, or $\gamma=0$, where the beams are incident on the side of the shaft. Notably, the geometric arrangements that allow measurement of the pitch and yaw vibration sets from the side of the shaft are independent of the angle $\beta$. With $\gamma=\frac{\pi}{2}, \beta= \pm \frac{\pi}{2}$ and $\alpha=0$ or $\gamma=0$ and $\alpha=\frac{\pi}{2}$, the pitch vibration set can be isolated:

$\Delta U_{m}=d\left[\dot{\theta}_{x}+\Omega \theta_{y}\right]$

Similarly, with $\gamma=\frac{\pi}{2}, \beta= \pm \frac{\pi}{2}$ and $\alpha=\frac{\pi}{2}$ or $\gamma=0$ and $\alpha=0$, the yaw vibration set can be isolated: 
$\Delta U_{m}=d\left[\dot{\theta}_{y}-\Omega \theta_{x}\right]$

Note the sensitivity to both pitch and yaw in both of these outputs. This is an important problem that can only be addressed by post-processing the outputs and this is described in section 4.2.

\section{MEASUREMENTS USING MULTIPLE PAIRS OF PARALLEL LASER BEAMS}

In many practical situations access to the shaft is restricted and it is not physically possible to align the beams at the angles set out in the previous section to isolate the required vibration set. The requirement for just one or two distinct values of $\alpha$ or $\beta$, rather than the possibility to measure at any orientation is very restrictive. For example, with this restriction, measurement of unambiguous rotation speed cannot be achieved from the end faces of the shaft which are often the only areas of access. An alternative optical arrangement capable of measuring the shaft rotation speed, pitch and yaw vibration sets from a variety of angles is, therefore, desirable.

Section 2.2 concluded that the most sensible optical arrangement to use was a pair of parallel beams so that the measurement is immune to the axial and radial vibration sets. Therefore, it seems natural to consider using pairs of parallel beams in the search for a more versatile method of measuring the rotational vibration sets. In order to simplify the task, pairs of beams with equal separations will be considered.

Using the velocity measured by a single pair of beams, given by equation (10a), the measurements from two pairs of beams can be formulated. With the measured velocities from each pair of beams available as an electrical signal, the possibility to add or subtract the 
velocities exists conveniently. The sum, $\Sigma(\Delta U)$, and difference, $\Delta(\Delta U)$, in the measured velocities are:

$$
\begin{aligned}
\Delta(\Delta U)= & \Delta U_{m 1}-\Delta U_{m 2} \\
= & d\left(\left(S \gamma_{1} S \beta_{1} C \alpha_{1}-C \gamma_{1} S \alpha_{1}-S \gamma_{2} S \beta_{2} C \alpha_{2}+C \gamma_{2} S \alpha_{2}\right)\left[\dot{\theta}_{x}+\Omega \theta_{y}\right]\right. \\
+ & \left(S \gamma_{1} S \beta_{1} S \alpha_{1}+C \gamma_{1} C \alpha_{1}-S \gamma_{2} S \beta_{2} S \alpha_{2}-C \gamma_{2} C \alpha_{2}\right)\left[\dot{\theta}_{y}-\Omega \theta_{x}\right] \\
& \left.+\left(S \gamma_{1} C \beta_{1}-S \gamma_{2} C \beta_{2}\right)\left[\dot{\theta}_{z}+\Omega\right]\right) \\
\Sigma(\Delta U)= & \Delta U_{m 1}+\Delta U_{m 2} \\
= & d\left(\left(S \gamma_{1} S \beta_{1} C \alpha_{1}-C \gamma_{1} S \alpha_{1}+S \gamma_{2} S \beta_{2} C \alpha_{2}-C \gamma_{2} S \alpha_{2}\right)\left[\dot{\theta}_{x}+\Omega \theta_{y}\right]\right. \\
+ & \left(S \gamma_{1} S \beta_{1} S \alpha_{1}+C \gamma_{1} C \alpha_{1}+S \gamma_{2} S \beta_{2} S \alpha_{2}+C \gamma_{2} C \alpha_{2}\right)\left[\dot{\theta}_{y}-\Omega \theta_{x}\right] \\
& \left.+\left(S \gamma_{1} C \beta_{1}+S \gamma_{2} C \beta_{2}\right)\left[\dot{\theta}_{z}+\Omega\right]\right)
\end{aligned}
$$

where the subscripts denote the particular pair of beams and $S \alpha_{1}=\sin \alpha_{1}, C \alpha_{1}=\cos \alpha_{1}$ etc. While there are numerous geometric arrangements of the pairs of beams that would enable the desired vibration set to be measured, in practice the number of useful geometric arrangements is limited to those that can be reliably set-up with acceptable accuracy in angle measurement. The most convenient way to arrange the pairs of beams is symmetrically, with a plane bisecting the angle between the planes of each laser beam pair, either perpendicular to, or parallel to, the shaft rotation axis and with equal values of $\gamma$. With such arrangements, measurements from both the side and end faces of the shaft are still possible. The geometric values required to measure the vibration sets using two pairs of parallel beams are summarised in Table 1 and depicted in Figures 6a-c. 
Table 1 shows three basic beam configurations exist. In the first, $\gamma_{1}=\gamma_{2}=\frac{\pi}{2}$ and $\beta_{1}=-\beta_{2}=\beta$, which means the beams are incident on the side of the shaft. In the second, $\gamma_{1}=\gamma_{2}=\frac{\pi}{2}$ and $\beta_{1}=\pi-\beta_{2}=\beta$, which means the beams are incident on the end face of the shaft. As shown in Figures 6a-c, the desired angular vibration set is selected by choosing the appropriate value for $\alpha$, while measurement of the rotation speed set can be achieved with any value of $\alpha_{1}=\alpha_{2}=\alpha$. This means that, for the same physical arrangement, measurements of the rotation speed set and one of the angular vibration sets can be obtained simultaneously simply by choosing to take both the sum and the difference in the velocities measured by each individual pair of beams. In the third configuration, $\gamma_{1}=\gamma_{2}=0$ and $\beta_{1}=\beta_{2}=0$, the beams are incident on the side of the shaft at different axial locations. This arrangement enables simultaneous measurement of the pitch and yaw vibration sets by taking both the sum and the difference in the velocities measured by each individual pair of beams.

The theory presented in this paper has enabled all of the possible, useful geometric arrangements to be identified. These include the single arrangement used in a previous study of torsional and bending vibration measurement [8] in which two laser torsional vibrometers were used to assess bending vibration on a diesel engine crankshaft. In this previous study, simultaneous measurement of the yaw and rotation speed sets gave an indication of the bending motion of the crankshaft but resolution of the individual pitch and yaw motion was not possible. Resolution would first require simultaneous measurement of the pitch and yaw vibration sets followed by application of a technique developed to resolve individual vibration components in the similar area of radial vibration measurement [9]. This resolution process is also well suited to application to the bending vibration measurements and it is outlined in the following section. 


\section{MEASUREMENT OF BENDING VIBRATION AND RESOLUTION OF PITCH AND YAW MOTIONS}

\subsection{PITCH \& YAW METER}

In order to make simultaneous measurements of pitch and yaw vibration, eliminating the rotation speed set, a novel optical configuration was devised. The configuration shares basic features with the laser tiltmeter [2] and Figure 7 shows the beam arrangement which is designed for use on the end face of a shaft and incorporates three parallel laser beams. The beams are aligned with the shaft spin axis and the angular vibration derived by using interferometers to subtract optically the velocity measured by beams 1 and 2 for the pitch vibration set and beams 1 and 3 for the yaw vibration set.

\subsection{RESOLUTION OF VIBROMETER OUTPUTS}

From equations (12a\&b), simultaneous measurements of the pitch, $\dot{\Theta}_{x}(t)$, and yaw, $\dot{\Theta}_{y}(t)$, vibration sets yield:

$\dot{\Theta}_{x}(t)=\dot{\theta}_{x}+\Omega \theta_{y}$

$\dot{\Theta}_{y}(t)=\dot{\theta}_{y}-\Omega \theta_{x}$

It is possible to manipulate equations $(14 \mathrm{a} \& \mathrm{~b})$ to give the genuine angular vibration velocities $\dot{\theta}_{x}$ and $\dot{\theta}_{y}$ in terms of the measurable quantities $\dot{\Theta}_{x}(t), \dot{\Theta}_{y}(t)$ and $\Omega$ by integration of the vibrometer outputs. If $\Omega$ is considered constant then: 
$\dot{\theta}_{y}=\Omega \theta_{x}\left(t_{0}\right)+\Omega \int_{t_{0}}^{t} \dot{\Theta}_{x}(t) d t-\Omega^{2} \int_{t_{0}}^{t} \theta_{y} d t+\dot{\Theta}_{y}(t)$

By expressing the vibratory motion as a Fourier series of $M$ components, where the $m^{\text {th }}$ component $\theta_{y_{m}}$ has amplitude $A_{m}$, angular frequency $\omega_{m}$ and phase $\phi_{m}$, a relationship between angular vibration velocity and the integral of angular vibration displacement at any one frequency can be found.

$\left.\int \theta_{y} d t\right|_{\omega=\omega_{m}}=-\frac{A_{m}}{\omega_{m}} \cos \left(\omega_{m} t+\phi_{m}\right)=-\frac{\dot{\theta}_{y_{m}}}{\omega_{m}^{2}}$

Substituting equation (16) into equation (15) and evaluating for the $m^{\text {th }}$ component i.e. at $\omega=\omega_{m}$ gives:

$\dot{\theta}_{y_{m}}=\left.W\left(\omega_{m}\right)\left(\dot{\Theta}_{y}(t)+\Omega \int_{t_{0}}^{t} \dot{\Theta}_{x}(t) d t\right)\right|_{\omega=\omega_{m}}$

where

$W\left(\omega_{m}\right)=\frac{\omega_{m}^{2}}{\omega_{m}^{2}-\Omega^{2}}$

Similarly, considering the pitch vibration: 


$$
\dot{\theta}_{x_{m}}=\left.W\left(\omega_{m}\right)\left(-\dot{\Theta}_{x}(t)+\Omega \int_{t_{0}}^{t} \dot{\Theta}_{y}(t) d t\right)\right|_{\omega=\omega_{m}}
$$

Therefore, the genuine vibration velocity components can be resolved by evaluating the second bracketed terms of equations (17a\&c) and then weighting by the frequency dependent function $W\left(\omega_{m}\right)$. These operations form the post-processing technique used for the experimental verification and the crankshaft vibration measurement.

Inspection of equations $(17 \mathrm{a}, \mathrm{b} \& \mathrm{c})$ shows that the weighting term is infinite and the bracketed term is zero for synchronous vibrations, i.e. $\omega_{m}=\Omega$, which makes this technique unsuitable for synchronous vibration measurement. This also means that a gap in the resolved data at the synchronous frequency is unavoidable and a velocity-time trace cannot therefore be reconstructed. It is important to realise that this is not a limitation of the post-processing technique but a limitation on the use of LDV that occurs for both translational and rotational vibration measurements on rotors. At synchronous frequency, equations (14a\&b) are no longer independent and cannot be solved as simultaneous equations.

Further work is in progress to improve the estimates of $\dot{\theta}_{x}$ and $\dot{\theta}_{\mathrm{y}}$ for the case where $\Omega$ is not constant, for example, when a system has significant torsional vibration or during run-up and run-down.

\subsection{EXPERIMENTAL VERIFICATION}

An extensive verification of equations (14a\&b) has already been reported [14] in which pitch and yaw motions were excited separately and the effect on the output from a dual beam laser vibrometer examined. Limiting the target motion to a single component minimises the complexity of the test rig required and removes the need for post-processing. Excellent 
agreement was found with theory. In what follows, the experimental verification is taken to the next stage with pitch and yaw motions excited simultaneously and post-processing therefore essential. The test rig shown in Figure 8 creates the required simultaneous angular vibrations of a small test rotor in two orthogonal directions. Measurements of angular vibration were made directly from the rotor, using the laser instrument described in section 4.1, and from the non-rotating motor housing by subtracting the outputs of two accelerometers separated by a known distance.

The pitch and yaw halves of the laser instrument were calibrated, one at a time, by making the beams incidents on a small test shaft, rotating at (nominally) constant speed, with the plane of the beams perpendicular to the shaft rotation axis. A very convenient independent measurement of angular velocity is provided by the pseudo-random noise in the instrument output caused by the laser speckle phenomenon [15]. Voltage output per unit angular velocity was thus reliably provided.

Two sets of measurements were made; firstly, with the rotor rotating at a nominal speed of $20 \mathrm{~Hz}$, then repeated with the rotor non-rotating. Figures 9a\&b show the ratio of the resolved laser and accelerometer measurements for the rotating measurements in the two orthogonal directions, normalised by the equivalent non-rotating measurements. Measurements of one angular vibration amplitude (nominally 50mrad/s) were combined with four phase differences $\left(0^{\circ}, \pm 90^{\circ}, 180^{\circ}\right)$ and eight vibration frequencies $(10,2530,40,60,80,100$ and $120 \mathrm{~Hz})$ with the mean and standard deviation of the ratio at each vibration frequency presented in the figures.

In the non-rotating tests, the outputs from the pitch and yaw meter are straightforward to interpret and so these measurements give an indication of the genuine differences between the different points on the structure at which the laser based and accelerometer measurements were taken. In the rotating tests, the laser instrument outputs need to be post-processed with 
agreement between the measurements now acting as validation of the theory leading to equations (14a\&b) and the post-processing method itself.

The resolved data from the rotating tests show good agreement with those from the nonrotating tests with the differences attributed to genuine differences between the vibration response of the rotor assembly when rotating and not rotating. It is, of course, the very existence of these genuine differences that is the motivation behind the development of techniques for measurement directly from the rotor. It should be emphasised that the comparison involves measurements taken by necessity from different locations since the accelerometer obviously cannot be attached to the rotor. This compounds the difficulty in finding the right data for comparison with the measurements from the pitch and yaw meter. A particular feature worth special mention is the deviation of values close to the rotation frequency $(20 \mathrm{~Hz})$ which can be linked to an increase in the sensitivity of the resolution process, through $W\left(\omega_{m}\right)$, to errors in the measurement of the shaft rotation speed [9].

\subsection{DIESEL ENGINE CRANKSHAFT VIBRATION}

The pitch and yaw meter was aligned parallel to the crankshaft rotation axis of a four cylinder two litre diesel engine and incident on the end face of the crankshaft pulley to provide simultaneous measurements of the pitch and yaw vibration sets. In addition a LTV was used to measure the crankshaft rotation speed. The beams of the LTV were aligned as close to perpendicular to the rotation axis as access would allow to eliminate the sensitivity of that measurement to bending vibration.

Figures $10 \mathrm{a} \& \mathrm{~b}$ show waterfall plots of the resolved crankshaft pitch and yaw vibration respectively across a range of engines speeds, obtained by varying engine load while maintaining wide open throttle. The broad peak in Figure 10a around $450 \mathrm{~Hz}$ corresponds to the first bending mode of the crankshaft confirmed using modal testing of the stationary 
crankshaft [8]. These are the first known resolved measurements of crankshaft pitch and yaw to be taken on a running engine by non-contact means and without modification to the crankshaft.

\section{CONCLUSIONS}

This paper has presented the first comprehensive theory to describe the velocity sensed by multiple laser beams incident on a rotating structure requiring three translational and three rotational co-ordinates to describe its vibratory motion fully. The theory is equally applicable to measurements on targets with simpler motions such as non-rotating targets, allowing the vibration engineer to determine the vibration component sensitivity for any measurement with any arrangement of beams.

Six separate vibration sets, each a combination of motion parameters, were identified in a previous study of vibration velocity sensitivity [7] which concluded that only the translational vibration sets could be isolated using a single laser beam. This paper extends this theory to include multiple beam arrangements which can isolate the rotational vibration sets and highlights the usefulness of parallel beam arrangements, demonstrating their insensitivity to the translational vibration sets. A concise new theory describing the difference velocity sensitivity for two parallel laser beams was presented and the geometric set-ups for measurement of the pitch, yaw and rotation speed sets established. A full range of versatile arrangements using two pairs of parallel beams was also presented as an alternative means to measure the pitch, yaw and rotation speed sets.

Resolution of individual motion components within each vibration set has been shown not to be possible by any arrangement of laser beams [7] so the use of a post-processing technique originally developed to resolve steady state, non-synchronous radial measurements [9] was extended to this application. This enabled the genuine pitch and yaw vibrations to be 
estimated from the measurements of the pitch and yaw vibration sets and the technique was validated experimentally in the laboratory.

Application of the measurement and resolution of pitch and yaw vibration was then applied to the assessment of bending vibration in the crankshaft of a running diesel engine enabling, amongst other features, identification of the first natural frequency of the crankshaft in bending. As the first such measurements to be taken by non-contact means, the potential of laser technology for machinery diagnostics under challenging conditions is, once again, emphasised.

\section{ACKNOWLEDGEMENTS}

J.R. Bell wishes to acknowledge the support of the Engineering and Physical Sciences Research Council. 


\section{REFERENCES}

1. D.C. WILLIAMS (ed.) 1993 Optical methods in engineering metrology Chapman \& Hall, London, Chapter 6.

2. N.A. HALLIWELL, A HOCKNELL and S.J. ROTHBERG 1997, Journal of Sound and Vibration 208(3) 497-500. On the measurement of angular vibration displacements: a laser tiltmeter.

3. M.W. TRETHEWEY, H.J. SOMMER and J.A. CAFEO 1993 Journal of Sound and Vibration 164(1) 67-84. A dual beam laser vibrometer for measurement of dynamic structural rotations and displacements.

4. A.B. STANBRIDGE and D.J. EWINS 1996 Shock and Vibration 3(2) 141-152. Measurement of translational and angular vibration using a scanning laser Doppler vibrometer.

5. N.A. HALLIWELL, C.J.D. PICKERING and P.G. EASTWOOD 1984 Journal of Sound and Vibration 93(4), 588-592. The laser torsional vibrometer: a new instrument.

6. T. KAMIYA, T. ATSUMI and K. TASAKA 1988 SAE Transactions 97(4) 4.59-4.65 SAE Paper 880078. Toyota's new type of crankshaft pulley to improve the compartment tone quality.

7. J.R. BELL and S.J. ROTHBERG 1999 submitted to Journal of Sound and Vibration. Laser vibrometers and contacting transducers, target rotation and 6 degree-of-freedom vibration: what do we really measure? 
8. T.J. MILES, M. LUCAS, N.A. HALLIWELL and S.J. ROTHBERG 1999 Journal of Sound and Vibration 226(3) 441-467. Torsional and bending vibration measurement on rotors using laser technology.

9. J.R. BELL and S.J. ROTHBERG 1998 Proceedings of SPIE - 3rd International Conference on Vibration Measurements by Laser Techniques: Advances and Applications, Ancona, Italy 3411 14-22. Radial vibration measurements on rotors using laser vibrometry: a first practical solution to the cross-sensitivity problem.

10. POLYTEC Rotational vibrometer - product data booklet.

11. N.A. HALLIWELL, L. PULLEN and J. BAKER 1983 SAE Special Publication 831324 986-994. Diesel engine health: laser diagnostics.

12. T. EIJU and K. MATSUDA 1991 Optical Engineering 30(11) 1825-1829. Determination of the central position of rotation of a rotating object by laser Doppler velocimetry.

13. J.H. WILLIAMS 1996 Fundamentals of applied dynamics Wiley \& Sons, New York.

14. T.J. MILES, M. LUCAS and S.J. ROTHBERG 1995 Proceedings of the 15th ASME Biennial Conference on Vibration and Noise, Boston, USA, 84-3(C) 1451-1460. The laser torsional vibrometer: successful operation during lateral vibrations.

15. S.J. ROTHBERG, J.R. BAKER and N.A. HALLIWELL 1989 Journal of Sound and Vibration 135(3) 516-522. Laser vibrometry: pseudo-vibrations. 


\begin{tabular}{|c|c|c|c|c|c|c|c|c|}
\hline $\begin{array}{c}\text { Desired } \\
\text { Measurement }\end{array}$ & Signal & $\alpha_{1}$ & $\alpha_{2}$ & $\beta_{1}$ & $\beta_{2}$ & $\gamma_{1}=\gamma_{2}$ & $\begin{array}{l}\text { Side or end } \\
\text { of shaft? }\end{array}$ & Figure \\
\hline \multirow{4}{*}{$\dot{\theta}_{x}+\Omega \theta_{y}$} & $\Delta(\Delta U)$ & 0 & 0 & $\beta$ & $-\beta$ & $\pi / 2$ & Side & $6 a$ \\
\hline & $\Sigma(\Delta U)$ & 0 & 0 & $\beta$ & $\pi-\beta$ & $\pi / 2$ & End & $6 a$ \\
\hline & $\Delta(\Delta U)$ & $\alpha$ & $-\alpha$ & 0 & 0 & 0 & Side & $6 c$ \\
\hline & $\Sigma(\Delta U)$ & $\alpha$ & $\pi-\alpha$ & 0 & 0 & 0 & Side & $6 c$ \\
\hline \multirow{4}{*}{$\dot{\theta}_{y}-\Omega \theta_{x}$} & $\Delta(\Delta U)$ & $\pi / 2$ & $\pi / 2$ & $\beta$ & $-\beta$ & $\pi / 2$ & Side & $6 \mathrm{~b}$ \\
\hline & $\Sigma(\Delta U)$ & $\pi / 2$ & $\pi / 2$ & $\beta$ & $\pi-\beta$ & $\pi / 2$ & End & $6 b$ \\
\hline & $\Sigma(\Delta U)$ & $\alpha$ & $-\alpha$ & 0 & 0 & 0 & Side & $6 c$ \\
\hline & $\Delta(\Delta U)$ & $\alpha$ & $\pi-\alpha$ & 0 & 0 & 0 & Side & $6 c$ \\
\hline \multirow[b]{2}{*}{$\dot{\theta}_{z}+\Omega$} & $\Sigma(\Delta U)$ & $\alpha$ & $\alpha$ & $\beta$ & $-\beta$ & $\pi / 2$ & Side & $6 a \& b$ \\
\hline & $\Delta(\Delta U)$ & $\alpha$ & $\alpha$ & $\beta$ & $\pi-\beta$ & $\pi / 2$ & End & $6 a \& b$ \\
\hline
\end{tabular}

Table 1: Configuration for measurement of pitch, yaw and rotation speed sets. 


\section{ROTATIONAL VIBRATION MEASUREMENT USING LASER DOPPLER \\ VIBROMETRY: COMPREHENSIVE THEORY AND PRACTICAL APPLICATION}

\section{J.R. BELL and S.J. ROTHBERG}

\section{FIGURES}

Figure 1. Definition of a point $\mathrm{P}$ on a vibrating and rotating component undergoing arbitrary vibration.

Figure 2. $\quad$ Orientation of laser beam, defining incident angles $\alpha$ and $\beta$.

Figure 3. “Cross-beam velocimeter” measurement.

Figure 4. Change in position vectors caused by target motion and shape variation.

Figure 5. Orientation of parallel beam separation.

Figure 6. Configurations of pairs of parallel laser beams for measurement of:

a. pitch and rotation speed sets.

b. yaw and rotation speed sets.

c. pitch and yaw sets.

Figure 7. New pitch and yaw meter beam arrangement.

Figure 8. Test rig to create simultaneous pitch and yaw motions.

Figure 9. Comparison of laser and accelerometer based measurements.

a. Measured pitch velocity, mean \pm 1 standard deviation.

b. Measured yaw velocity, mean \pm 1 standard deviation.

Figure 10. Diesel engine crankshaft bending vibration

a. Resolved pitch vibration.

b. Resolved yaw vibration. 


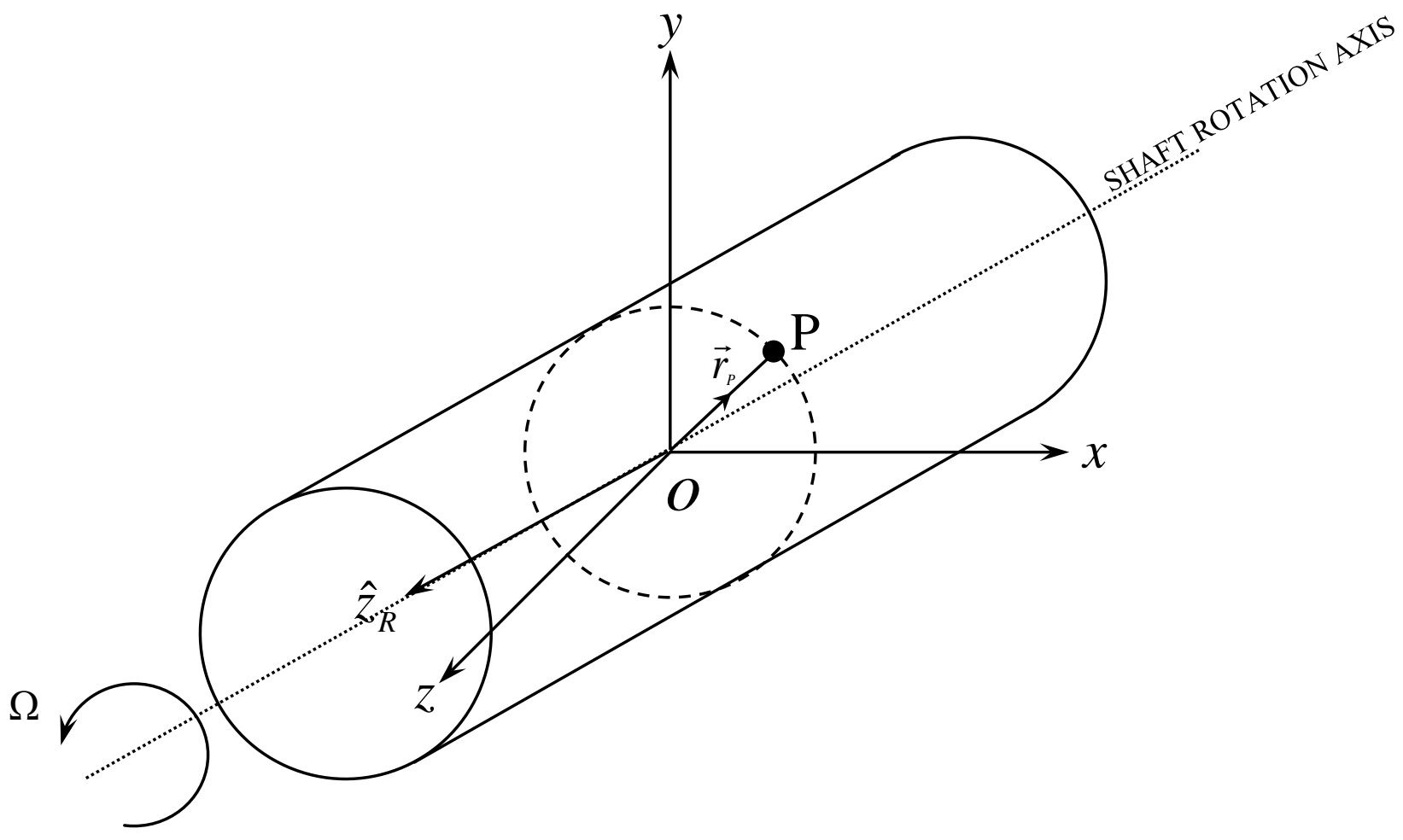

Figure 1

Rotational Vibration Measurement using Laser Doppler Vibrometry:

Comprehensive Theory and Practical Application

J.R. Bell and S.J. Rothberg 


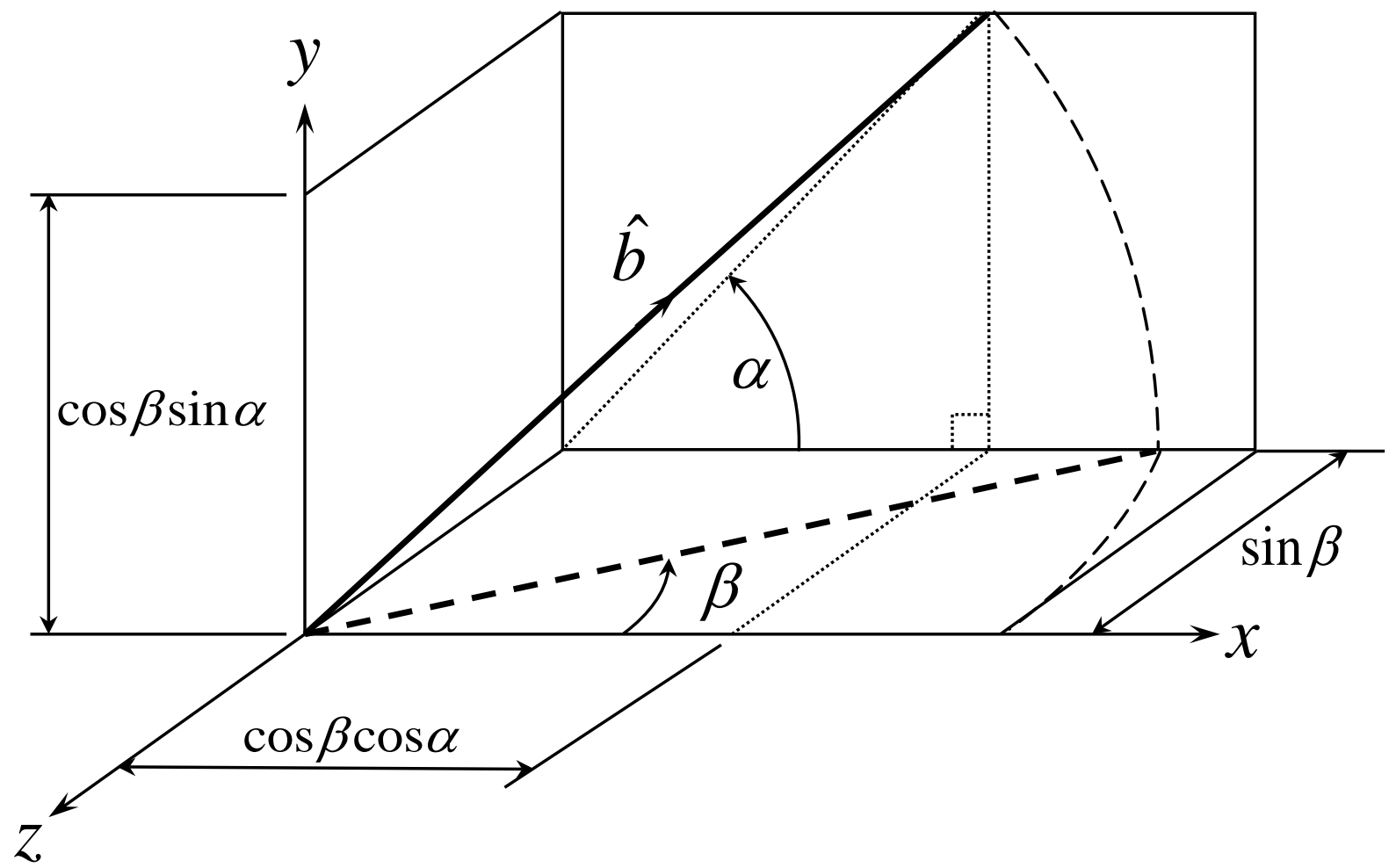

Figure 2

Rotational Vibration Measurement using Laser Doppler Vibrometry:

Comprehensive Theory and Practical Application

J.R. Bell and S.J. Rothberg

Loughborough University 


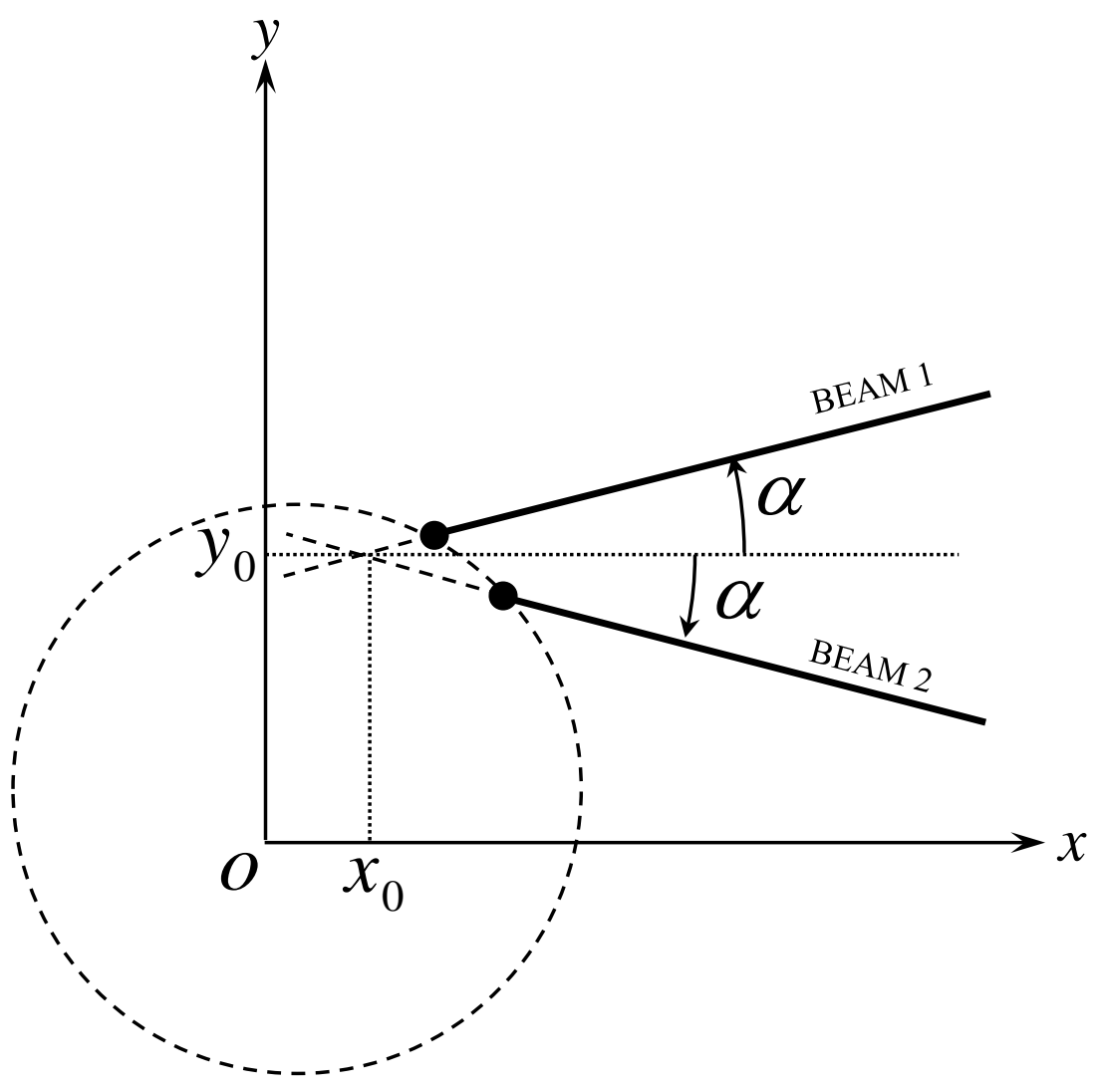

Figure 3

Rotational Vibration Measurement using Laser Doppler Vibrometry:

Comprehensive Theory and Practical Application

J.R. Bell and S.J. Rothberg

Loughborough University 


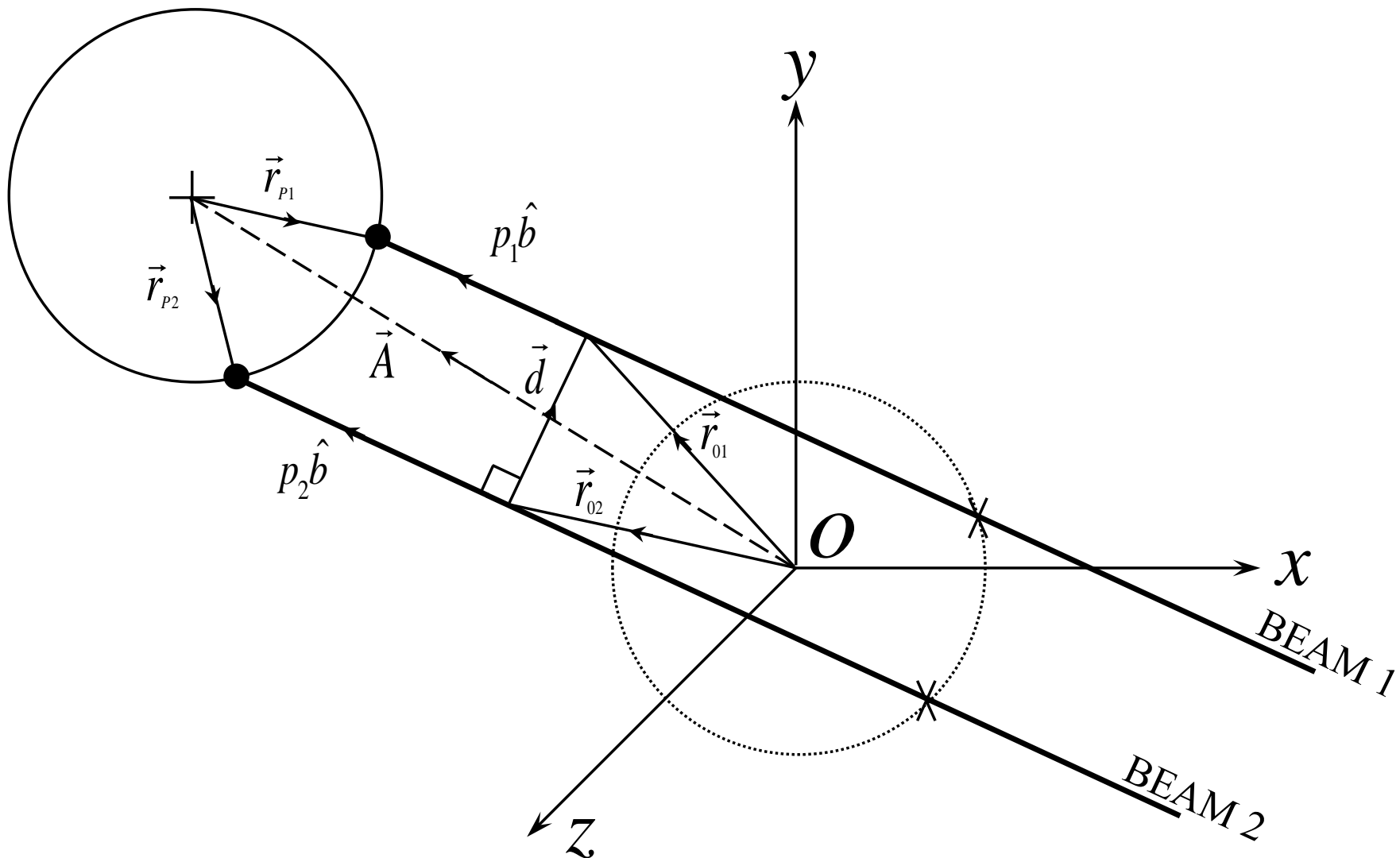

Figure 4

Rotational Vibration Measurement using Laser Doppler Vibrometry:

Comprehensive Theory and Practical Application

J.R. Bell and S.J. Rothberg 


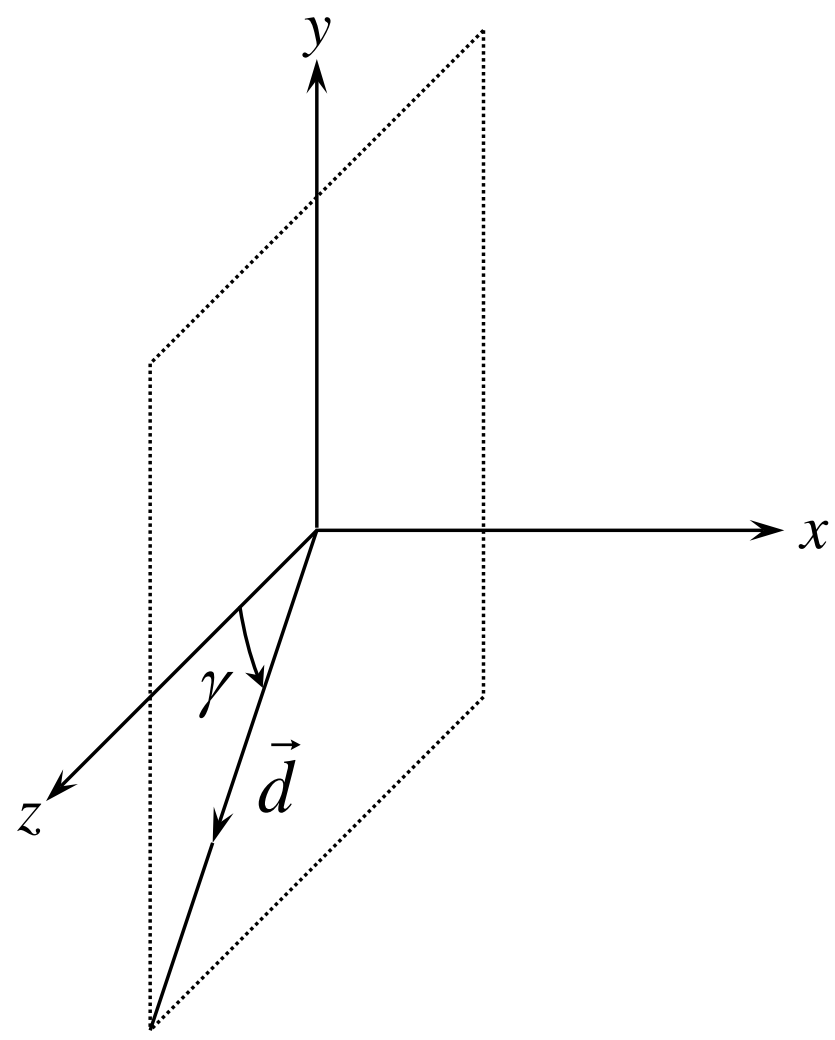

Figure 5

Rotational Vibration Measurement using Laser Doppler Vibrometry:

Comprehensive Theory and Practical Application

J.R. Bell and S.J. Rothberg

Loughborough University 


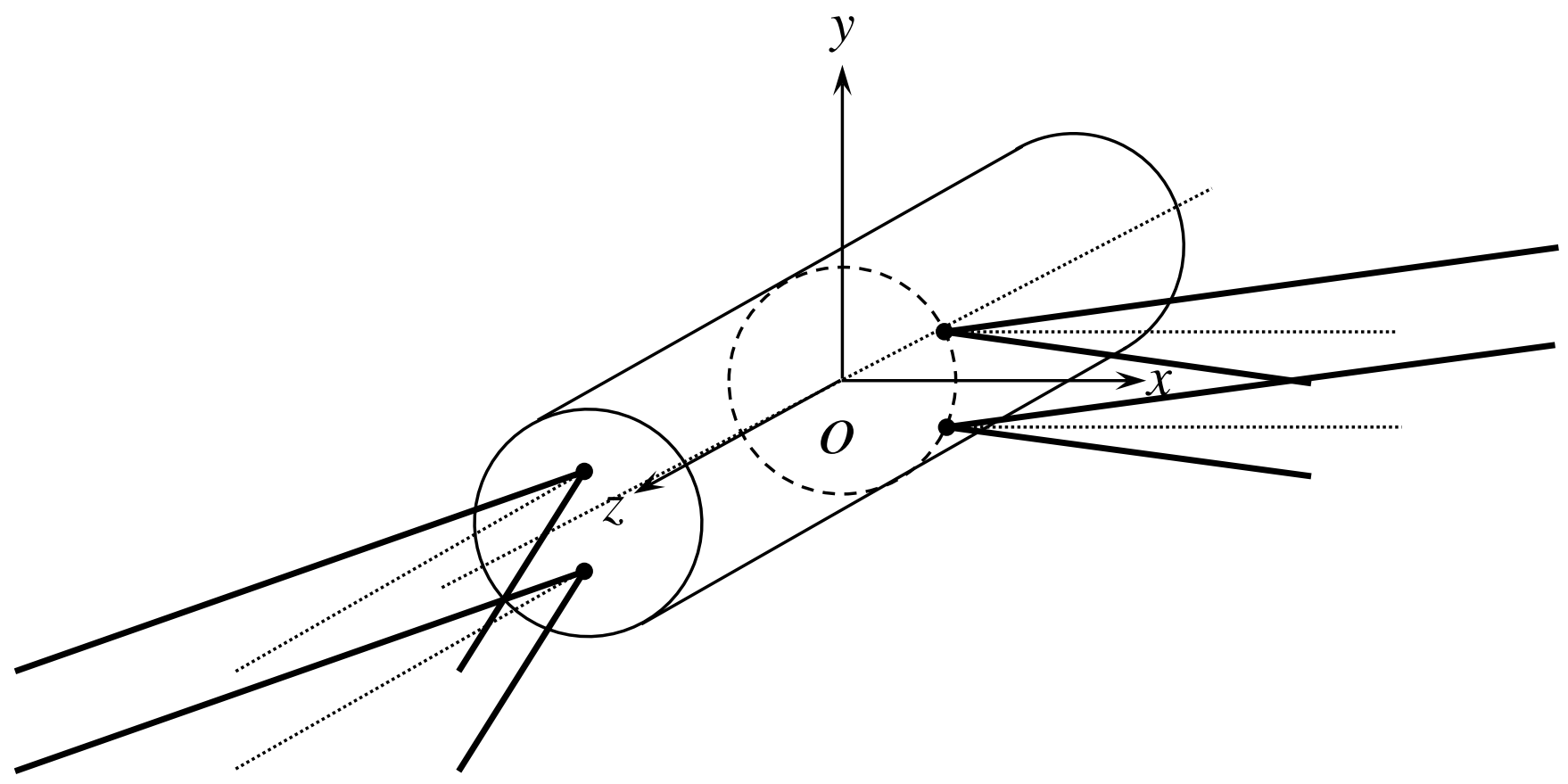

Figure 6a

Rotational Vibration Measurement using Laser Doppler Vibrometry:

Comprehensive Theory and Practical Application

J.R. Bell and S.J. Rothberg

Loughborough University 


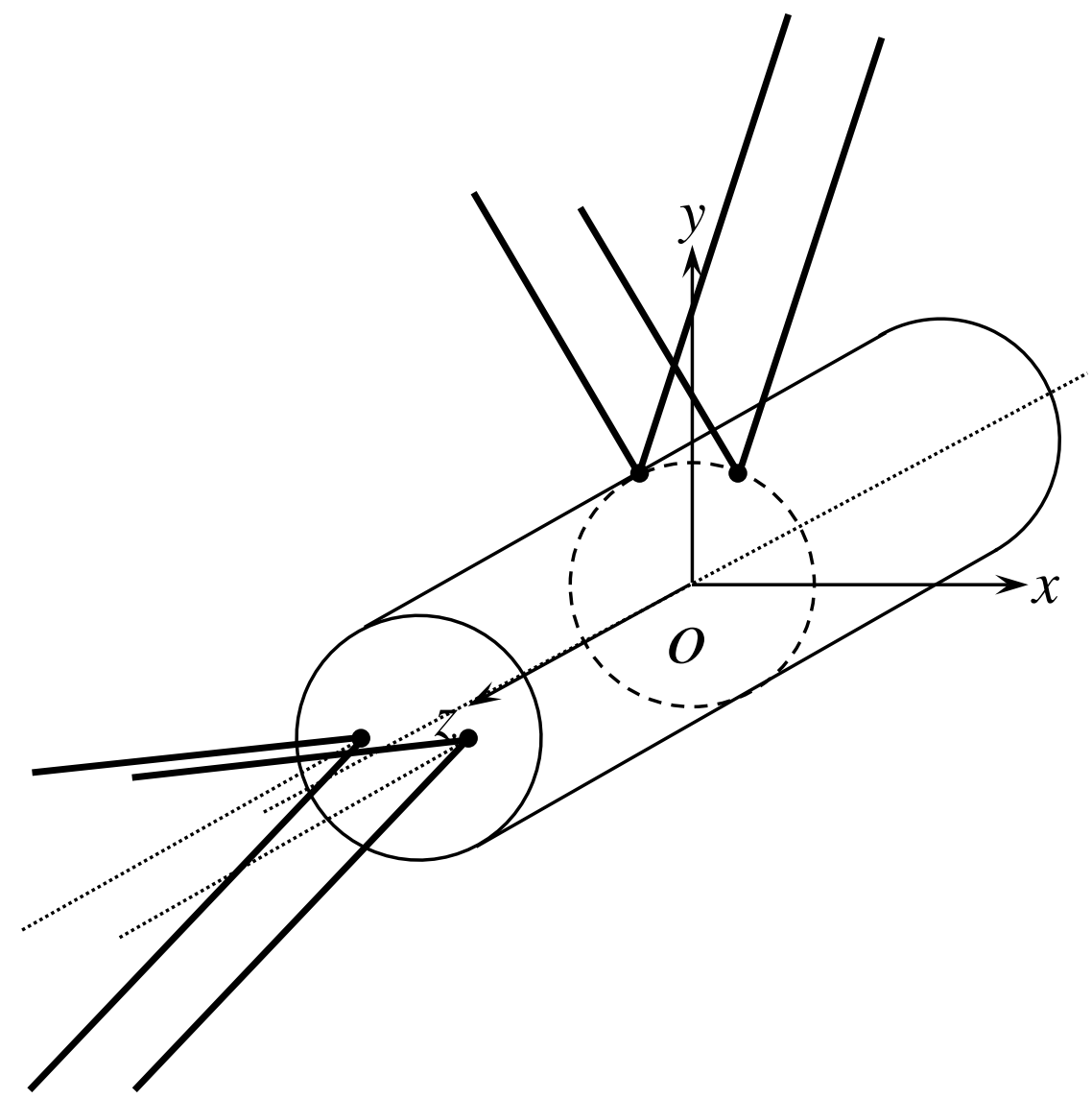

Figure 6b

Rotational Vibration Measurement using Laser Doppler Vibrometry:

Comprehensive Theory and Practical Application

J.R. Bell and S.J. Rothberg

Loughborough University 


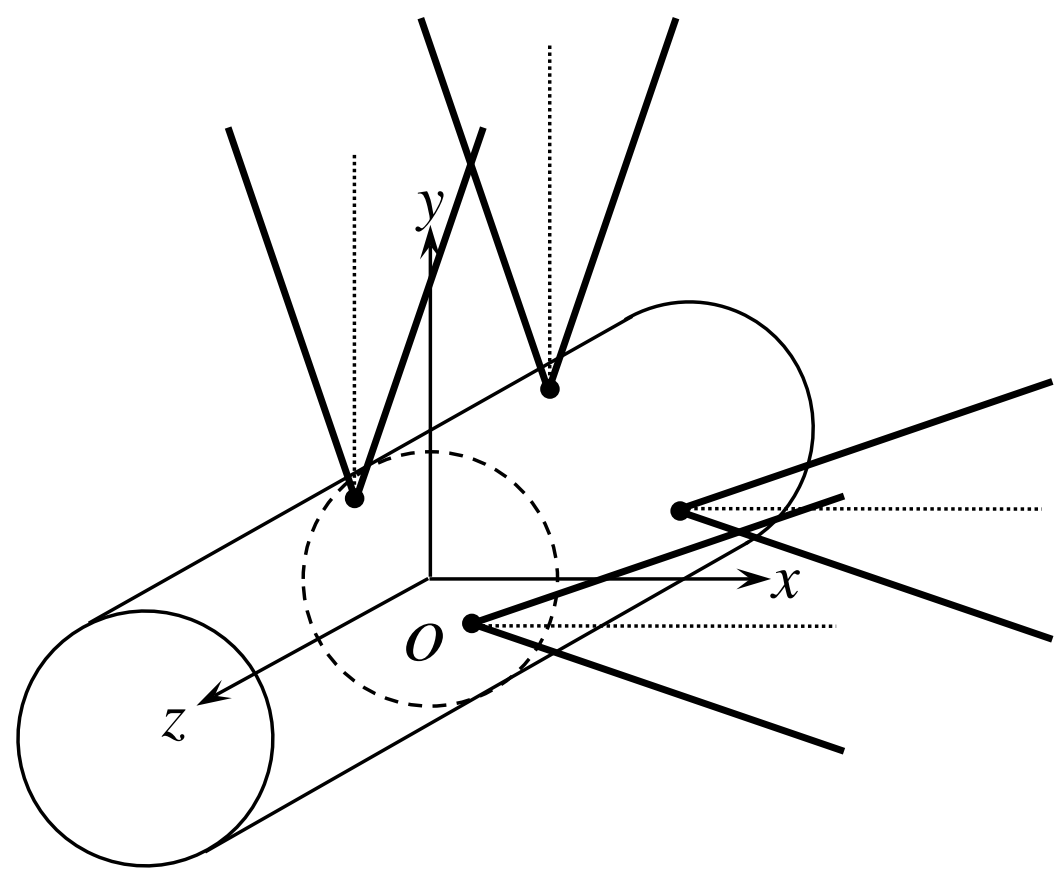

\section{Figure 6c}

Rotational Vibration Measurement using Laser Doppler Vibrometry:

Comprehensive Theory and Practical Application

J.R. Bell and S.J. Rothberg 


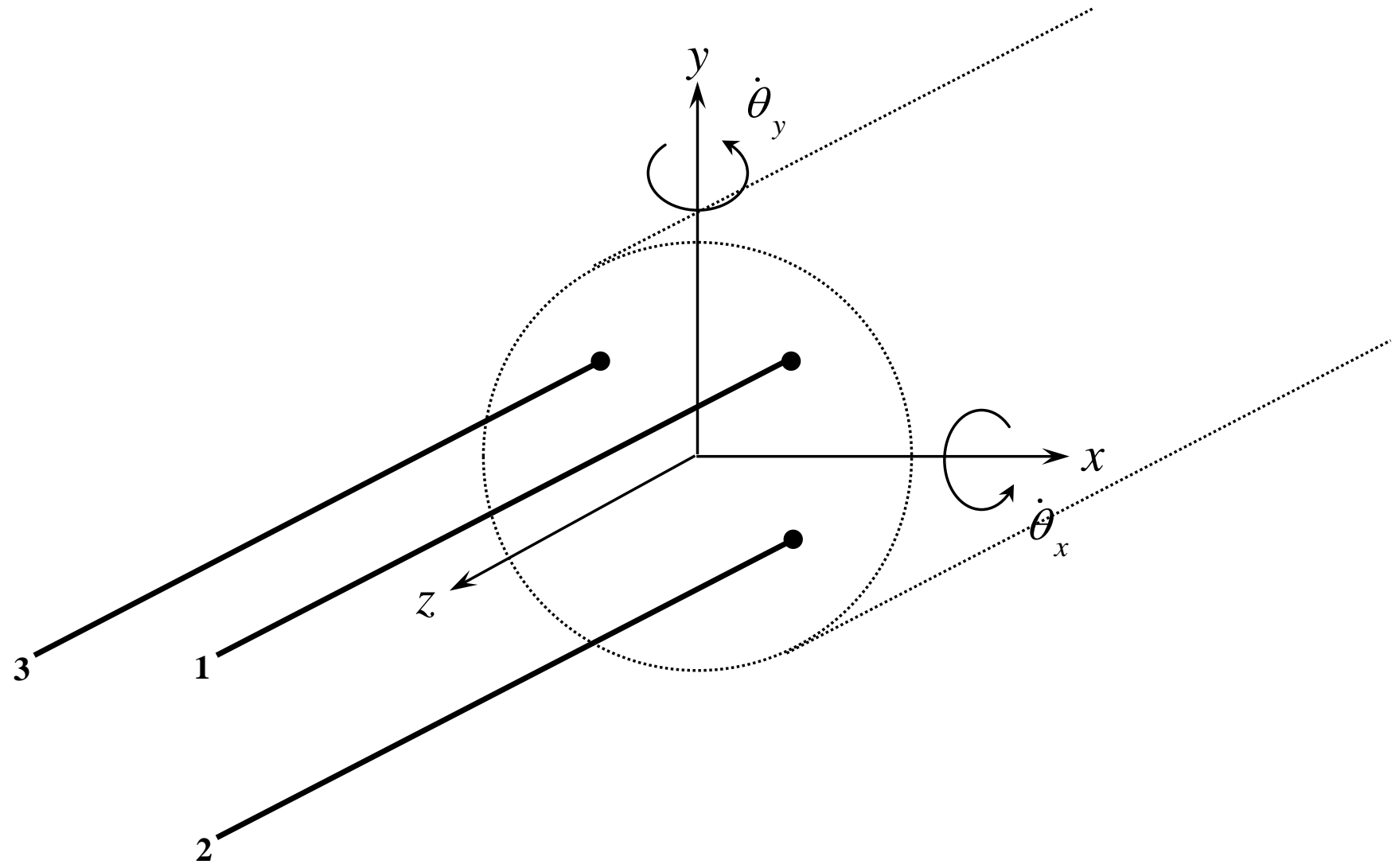

Figure 7

Rotational Vibration Measurement using Laser Doppler Vibrometry:

Comprehensive Theory and Practical Application

J.R. Bell and S.J. Rothberg

Loughborough University 


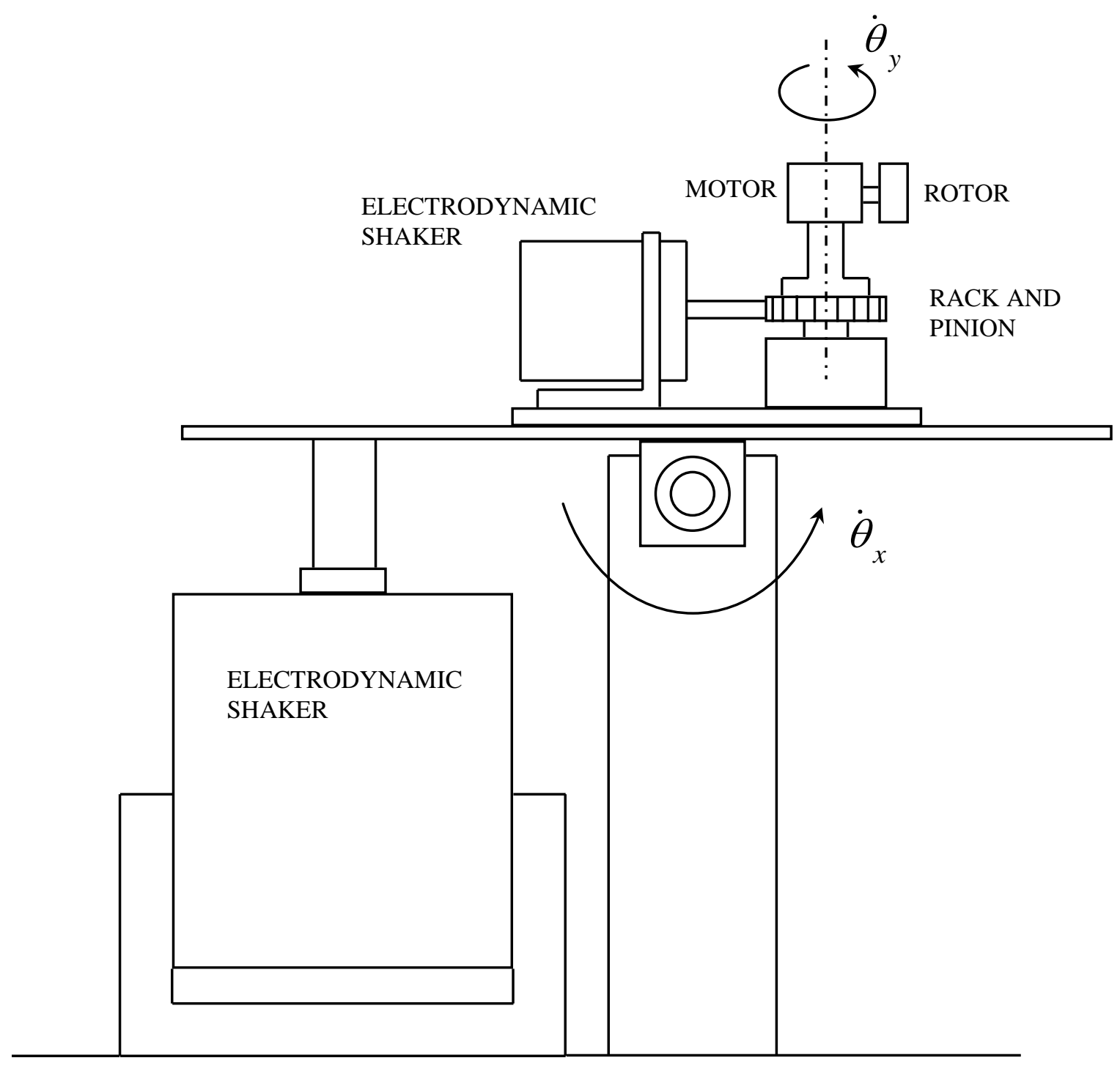

Figure 8

Rotational Vibration Measurement using Laser Doppler Vibrometry:

Comprehensive Theory and Practical Application

J.R. Bell and S.J. Rothberg 


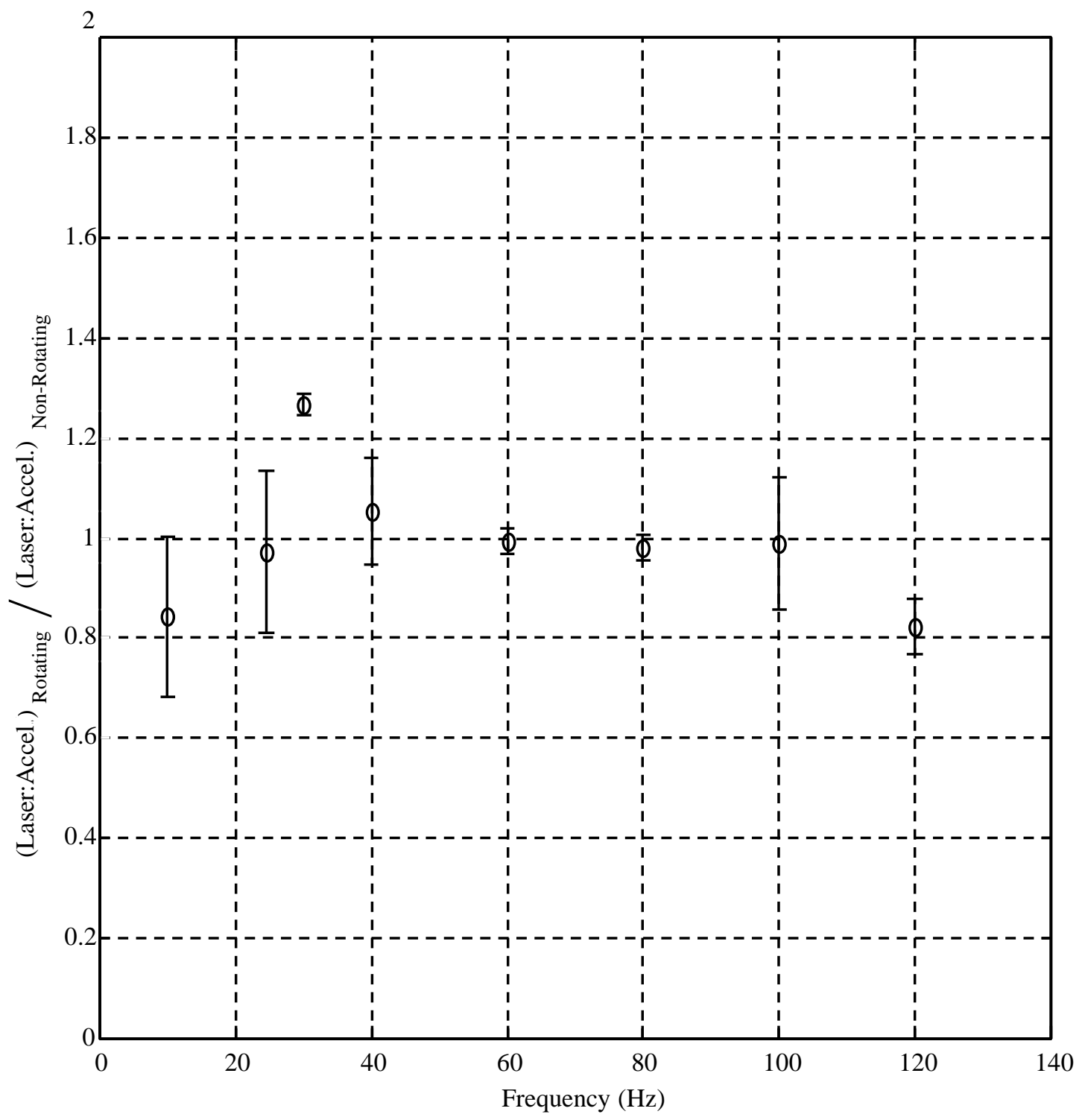

Figure 9a

Rotational Vibration Measurement using Laser Doppler Vibrometry: Comprehensive Theory and Practical Application

J.R. Bell and S.J. Rothberg 


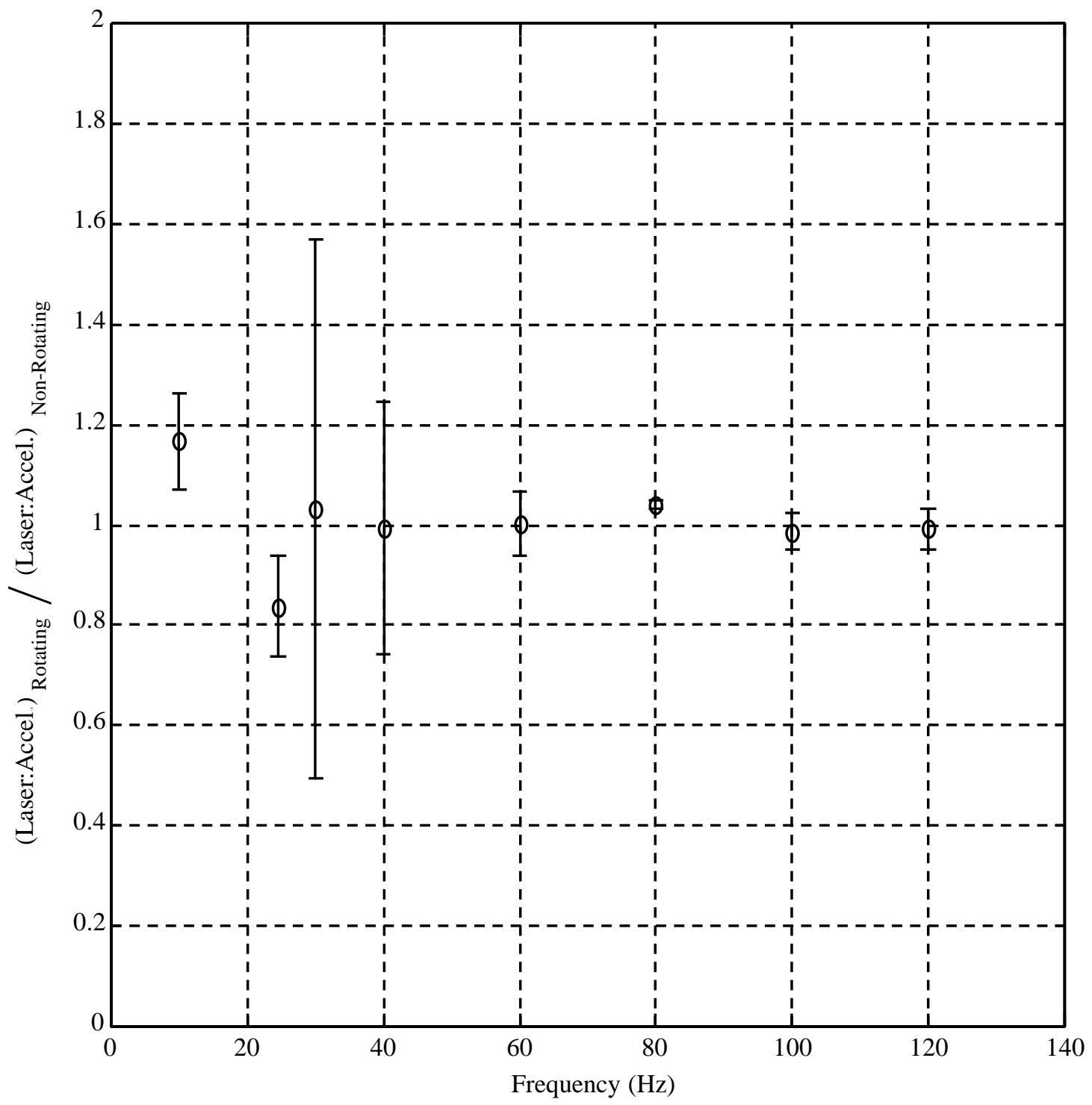

Figure $9 \mathrm{~b}$

Rotational Vibration Measurement using Laser Doppler Vibrometry: Comprehensive Theory and Practical Application 


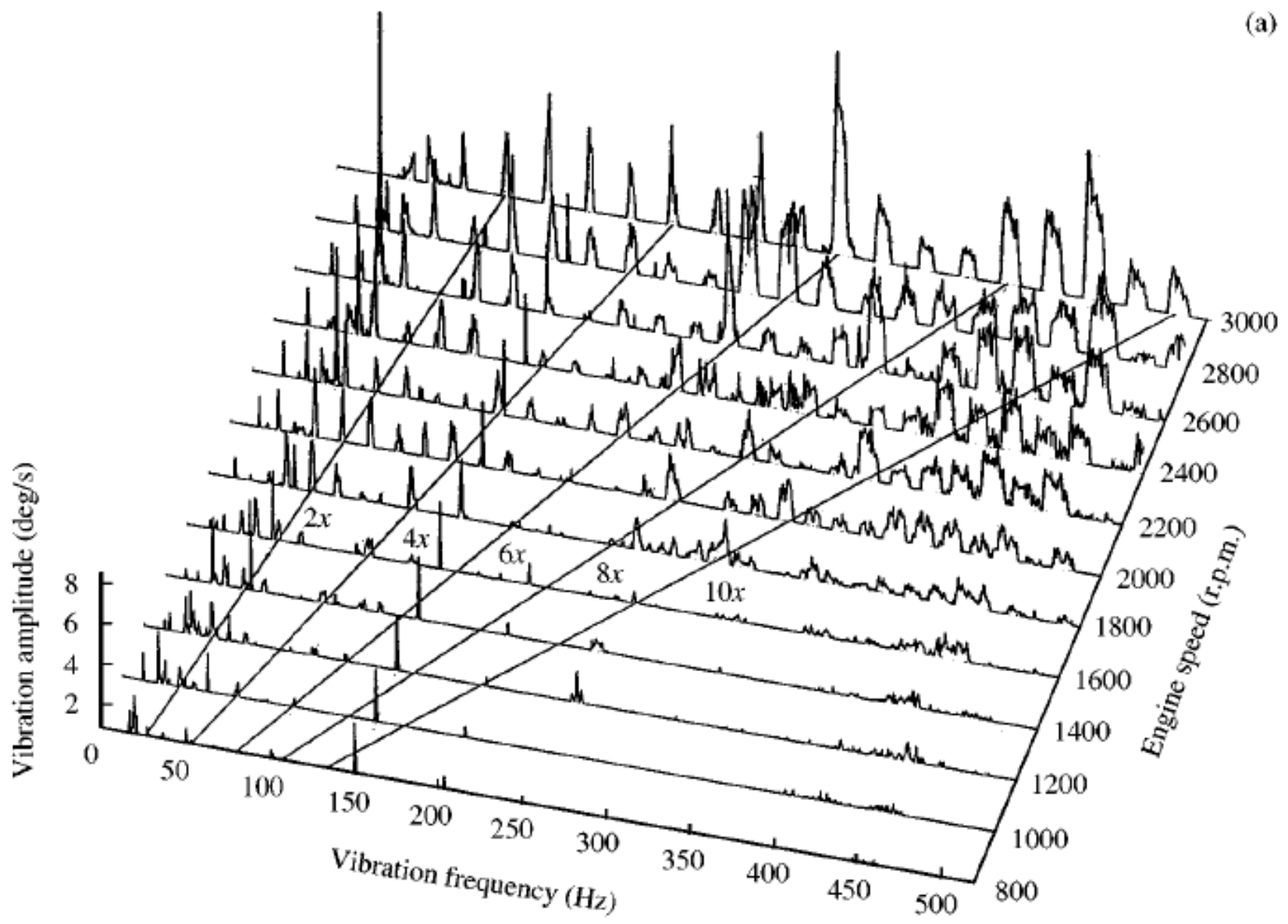

Figure 10a

Rotational Vibration Measurement using Laser Doppler Vibrometry: Comprehensive Theory and Practical Application 


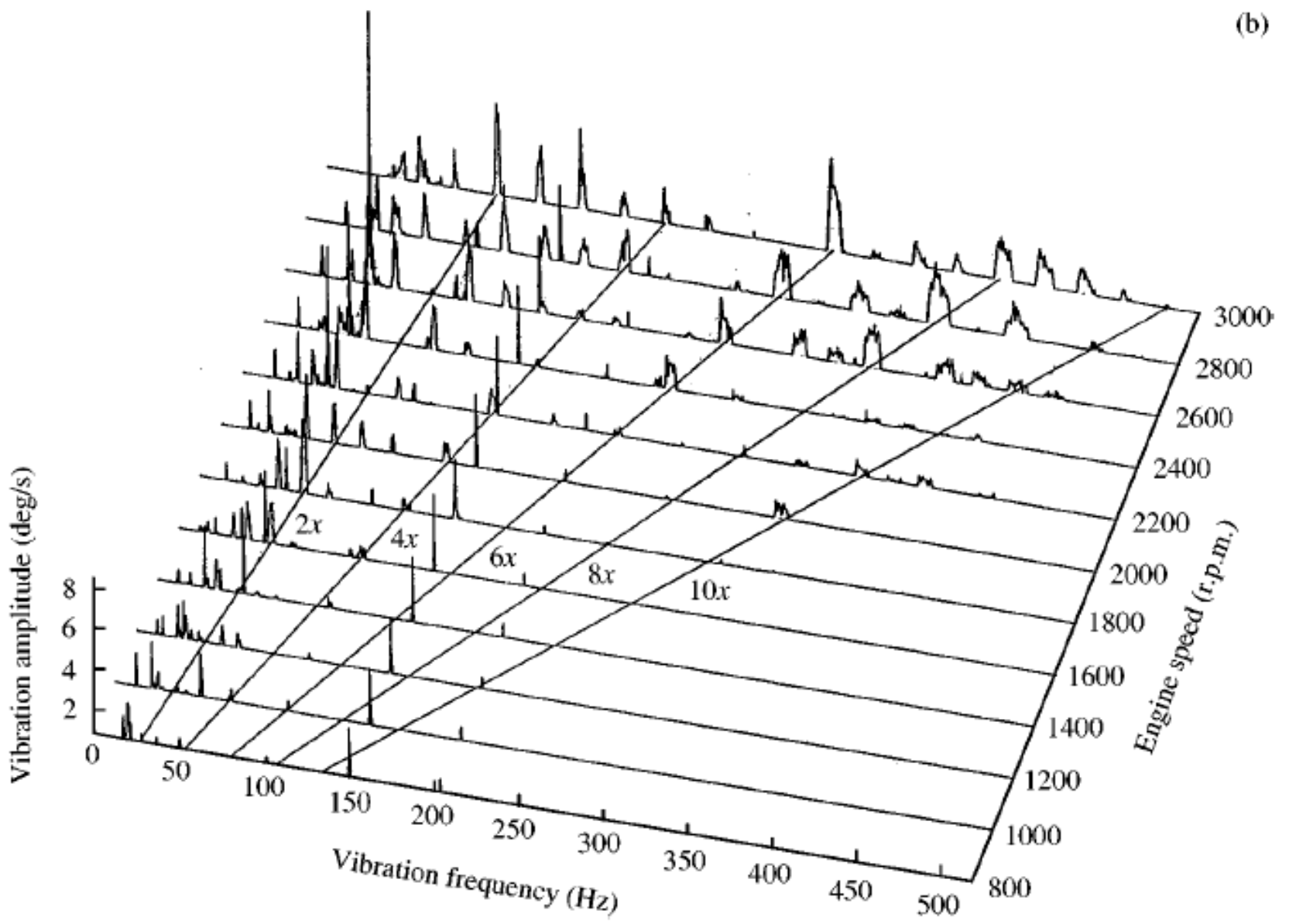

Figure 10b

Rotational Vibration Measurement using Laser Doppler Vibrometry: Comprehensive Theory and Practical Application

J.R. Bell and S.J. Rothberg 